Preface

\title{
Circadian, sleep-wake dependent or both? A preface to the special issue "Circadian rhythm and sleep-wake dependent regulation of behavior and brain function"
}

\section{Introduction}

Life has evolved around the Earth's day-night cycle. It is, therefore, not surprising that all living organisms, from bacteria to human beings, have a circadian system that sets their physiology in time over a near$24 \mathrm{~h}$ cycle, sometimes superimposed on other shorter (ultradian, circatidal) or longer (lunar, seasonal) cycles. The circadian system can be viewed as serving two fundamental roles. First, it allows organisms to anticipate environmental changes so that required functions are optimal when needed but are also quiet when not needed. Second, the circadian system prevents incompatible processes from occurring simultaneously. This leads to important energy savings over processes that would be very costly to run continuously or that would work against each other [1].

Research has found circadian regulation in most, if not all, physiological measures, as reflected for instance, in the fact that more than $80 \%$ of protein-coding genes show daily rhythms in expression in primate [2]. This includes, therefore, the brain, where the master circadian clock lies - within the hypothalamus suprachiasmatic nuclei - and the output of brain function, i.e., behavior [3]. Although this seems obvious, the circadian system often remains underestimated or ignored. Medicine is starting to acknowledge that symptoms and treatment responses depend on the time of assessment and the time of intervention. It is increasingly clear, for instance, that drug pharmacokinetics and pharmacodynamics exhibit substantial circadian or daily variations, including neurological or psychiatric disorder treatments $[1,4]$. Likewise, cognitive performance varies over the day and these variations change over the lifespan [5].

Apart from these circadian system aspects bearing important health and well-being implications, neuroscientists often neglect the variability of neurochemical concentrations, neuronal structure, and cognitive performance over $24 \mathrm{~h}$. If this $24 \mathrm{~h}$ variation is smaller than the effect being studied, the noise within the data will be increased, reducing the sensitivity of the experimental protocol for statistically detecting the effect. As a result, more observations, animals or patients will be needed. In contrast, if the $24 \mathrm{~h}$ variation is larger than the investigated effect, no statistical association can be detected between processes that are nevertheless associated. Accounting for circadian variation while evaluating a process of interest is, therefore, not just adding a layer of complexity, it may be the key to a successful experiment.

On top of this cycling circadian physiology, evolution has selected sleep as an obligatory phase in the animal kingdom. Sleep is a state of great vulnerability, requiring organisms to seek safe places or watch mechanisms to protect against predators and accidents. The functions of sleep are far from fully elucidated but they must be fundamental and also incompatible with wakefulness to put all animals in danger every day. The aim of this special issue is to report on some of the latest basic, clinical and translational research findings that address some of the knowledge gaps about the roles of circadian rhythms and sleep for optimal brain functioning, cognition and health.

Sleep is not only a matter of how long you've been awake, it is gated by the circadian system that can either facilitate or prevent sleep to occur so that, when sleep does occur is also crucially important [3]. Although intimately embedded in chronobiology, sleep research has often forgotten to register sleep in its circadian rhythmicity. This omission could be problematic as the interaction between sleep homoeostasis, keeping track of time awake, and building up sleep need, and the circadian system is non-linear [3]. During the day, a circadian signal progressively opposes sleep need to maintain stable behavior. At night, however, the circadian system changes roles and ceases opposing sleep need and may even turn into a sleep-favoring signal. This nonlinear interaction is among the main reasons why human errors and accidents are more prevalent at the end of the night when sleep need can be very high and the circadian system pushes us towards sleep. Nonlinearity also means that it is difficult to separate sleep from circadian functions. It further implies that controlling for a circadian impact cannot be truly achieved by testing all individuals at the same circadian phase or time, for instance, as it depends on prior sleep-wake history. This advocacy for considering both sleep homoeostasis and circadian regulation of physiology, in general, and of the brain, in particular, does not mean that focusing on one and not the other cannot provide important keys to sleep-wake regulation of brain function. Under some circumstances, it can be very complicated to consider them together. One should, however, always seek an ultimate integration of both and avoid minimizing the influence one process may have on the other. Importantly, concluding that a given process is circadian or sleep-wake dependent, or both, requires a thorough and appropriate test of their joint impacts.

This special issue starts with a commentary in line with this latter point. It considers the hypothesized synaptic function of sleep and argues that the contribution of the circadian system to the overnight daily synaptic scaling has not been fully assessed and quantified so that the real function of sleep for synapses cannot be completely characterized [10.1016/j.bcp.2021.114533]. Also emphasizing that sleep is not exclusively for the benefit of memory, the second paper $[10.1016 / \mathrm{j}$. bcp.2020.114369] is a review emphasizing that memory consolidation starts during wakefulness immediately after learning, before summarizing the evidence in favor of the role of sleep for memory and synaptic function [6], as a direct complement to the initial commentary. Also 
related to the initial commentary, the next review [10.1016/j.bcp.2021 114493] summarizes the roles of the so-called "clock genes" (i.e., circadian genes) in sleep, stress and memory [7], further reinforcing that physiological processes are intermingled.

The issue continues with an overview of the neurobiology of the circadian signal [insert DOI link to BP-D-20-01980/ Zeitzer] that gates sleep and wakefulness and argues that hypocretin/orexin may be the physiologic instantiation of the circadian wake drive [8]. This review is then complemented by an original research article [10.1016/j.bcp.2020 .114386] of the neurobiology of Rapid Eye Movement (REM) sleep [9]. The two following publications consider the joint impact of the circadian and sleep homoeostasis system on brain function and behaviour. The first one [10.1016/j.bcp.2021.114518] focuses on the human brain and the changes in brain response complexity during prolonged overnight wakefulness and overnight sleep loss [10]. Then the evidence in favour of the links between local sleep homoeostasis and circadian rhythmicity and mind wandering, which, intuitively, appears more likely when sleepiness is high [10.1016/j.bcp.2021.114478] is reviewed [11]. The next original report [10.1016/j.bcp.2020.114111] reminds us that, although dysfunctional during sleep loss, we are still able to perform, even if at a lower level, meaning that the brain is equipped to do without sleep for a while. The report tells us that one strategy to face sleep loss during learning can be to have appropriate interaction with a nearby well-rested instructor [12].

Next is a comparison of the age-related changes in the sleep and circadian rhythmicity in rodents and humans with obvious consistency, but also important differences, followed by a summary of part of the evidence in favor of physical activity intervention to maintain or restore sleep quality [insert DOI link to BP-D-21-00450/DeBoer]. In direct continuation of this review, the special issue turns to interventions. Four papers will deal with light as the main environmental cue for sleep and wakefulness regulation. Beyond its visual role, light signal bears many non-image-forming dimensions [3]. Light can stimulate alertness, attention and cognition. These impacts are further assessed using neuroimaging in patients with retinal degeneration [13] [10.1016/j.bcp.20 21.114488] and healthy individuals [14] [10.1016/j.bcp.2021.11450 4], together with light impact on melatonin secretion and circadian rhythmicity. The non-image-forming impact of light on memory has been difficult to assess given that long-term memory encompasses encoding, consolidation and retrieval and the tight links between attention and memory. The special issue includes an original research paper [10.1016/j.bcp.2020.114404] on the topic in mice, which are nocturnal and in which therefore light does not increase but rather decreases alertness [15]. The three basic papers on light are followed by a reflection on the importance of daylight in more ecological situations and advocates for the use of light as a drug for some diseases and disorders [16] [10.1016/j.bcp.2020.114304].

The special issue then shifts to chemical compounds. First, an original paper dealing [10.1016/j.bcp.2020.114283] with the impact of caffeine on sleep and circadian timing, with a focus on teenagers, for whom caffeine consumption may be particularly problematic given their already insufficient sleep [17]. Rather than using stimulants to fight sleepiness, one can use GABAergic compound, i.e., "sleeping pills", to induce sleep. An original research paper [10.1016/j.bcp.2021.114515] reports on the effect of diazepam on brain electrical signal in mice [18].

As in any field, mathematical models are precious tools to link, for instance, basic findings in animals to human disorders. The special issue includes two such papers. The first of which [10.1016/j.bcp.2021.11 4482] covers the use of models to gain understanding in sleep disorders and jet-lag based on basic clock mechanisms [19]. The second [10 .1016/j.bcp.2020.114388] deals with how the understanding of unconsciousness and anaesthesia has gained from mathematical modelling of how of the circadian and sleep homoeostasis processes regulating brain activity rhythms during sleep [20].

The special issue concludes with much more ecological/real-life considerations. A review [10.1016/j.bcp.2021.114438] focuses on how inappropriate social constraints on sleep-wake timing in different chronotypes may lead to circadian and sleep disruptions that can negatively impinge on cognition [21]. This overview of in-lab and more epidemiological findings is followed by an original study $[10.1016 / \mathrm{j}$. bcp.2021.114463] of a situation we all faced recently: what was the impact of COVID-19 lockdown on sleep quality? The study emphasizes that sleep quality is not only about circadian timing and allowing enough time for sleep: although most people went to bed later when they had more freedom regarding their schedules, sleep quality mostly worsened during the lockdown [22].

The content of this special issue may appear surprising in a journal about biochemistry and pharmacology. The aim is to enlarge the view of biochemistry and pharmacology researchers outside their typical focus of interest to appreciate how circadian system aspects may influence experimental outcomes. In turn, I hope chronobiologists and sleep neuroscientists will remember that brain function and behaviour are intimately entwined with interactions between biochemical compounds.

\section{Acknowledgments}

GV is supported by Fonds National de la Recherche Scientifique (FRS-FNRS Belgium ; PDR T.0242.19), ULiège (Easy), Federation of Wallonia and Brussels (Arction de Recherches concertées - ARC - 09/1403), Fondation Recherche Alzheimer (SAO-FRA 2019/0025).

\section{References}

[1] C.R. Cederroth, U. Albrecht, J. Bass, S.A. Brown, J. Dyhrfjeld-Johnsen, F. Gachon, C.B. Green, M.H. Hastings, C. Helfrich-Förster, J.B. Hogenesch, F. Lévi, A. Loudon, G.B. Lundkvist, J.H. Meijer, M. Rosbash, J.S. Takahashi, M. Young, B. Canlon, Medicine in the fourth dimension, Cell Metab. 30 (2019) 238-250, https://doi.org/ 10.1016/j.cmet.2019.06.019.

[2] L.S. Mure, H.D. Le, G. Benegiamo, M.W. Chang, L. Rios, N. Jillani, M. Ngotho, T. Kariuki, O. Dkhissi-Benyahya, H.M. Cooper, S. Panda, Diurnal transcriptome atlas of a primate across major neural and peripheral tissues, Science (80-. ). (2018) eaao0318. 10.1126/science.aao0318.

[3] G. Gaggioni, P. Maquet, C. Schmidt, D. Dijk, G. Vandewalle, Neuroimaging, cognition, light and circadian rhythms, Front. Syst. Neurosci. 8 (2014) 126, https://doi.org/10.3389/fnsys.2014.00126.

[4] G.A. FitzGerald, Temporal targets of drug action, Science (80-. ). 346 (2014) 921-922. 10.1126/science.aaa2285.

[5] C. Schmidt, P. Peigneux, C. Cajochen, Age-related changes in sleep and circadian rhythms: Impact on cognitive performance and underlying neuroanatomical networks, Front. Neurol. JUL (2012) 118, https://doi.org/10.3389/fneur.2012.00118.

[6] W. Stee, P. Peigneux, Post-learning micro- and macro-structural neuroplasticity changes with time and sleep, Biochem. Pharmacol. (2020), 114369, https://doi. org/10.1016/j.bcp.2020.114369.

[7] Y.G. Bolsius, M.D. Zurbriggen, J.K. Kim, M.J. Kas, P. Meerlo, S.J. Aton, R. Havekes, The role of clock genes in sleep, stress and memory, Biochem. Pharmacol. (2021), 114493, https://doi.org/10.1016/j.bcp.2021.114493.

[8] J.M. Zeitzer, The neurobiological underpinning of the circadian wake signal, Biochem. Pharmacol. (2020), 114386, https://doi.org/10.1016/j.bcp.2020.114386.

[9] R. Maciel, R. Yamazaki, D. Wang, A. De Laet, S. Cabrera, C. Agnorelli, S. Arthaud, P.-A. Libourel, P. Fort, H. Lee, C. Queiroz, P.-H. Luppi, Is REM sleep a paradoxical state?: Different neurons are activated in the cingulate cortices and the claustrum during W and paradoxical sleep hypersomnia, Biochem. Pharmacol. (2021), 114514, https://doi.org/10.1016/j.bcp. 2021.114514.

[10] G. Gaggioni, E. Shumbayawonda, U. Montanaro, J.Q.M. Ly, C. Phillips, G. Vandew Alle, D. Abásolo, Time course of cortical response complexity during extended wakefulness and its differential association with vigilance in young and older individuals, Biochem. Pharmacol. (2021), 114518, https://doi.org/10.1016/j. bcp.2021.114518.

[11] E. Jubera-Garcia, W. Gevers, F. Van Opstal, Local build-up of sleep pressure could trigger mind wandering: Evidence from sleep, circadian and mind wandering research, Biochem. Pharmacol. (2021), 114478, https://doi.org/10.1016/j. bcp.2021.114478.

[12] Y. Pan, C. Guyon, G. Borragán, Y. Hu, P. Peigneux, Interpersonal brain synchronization with instructor compensates for learner's sleep deprivation in interactive learning, Biochem. Pharmacol. (2020), 114111, https://doi.org/10.1016/j. bcp. 2020.114111 .

[13] S. Evangelisti, C. La Morgia, C. Testa, D.N. Manners, L. Brizi, C. Bianchini, M. Carbonelli, P. Barboni, A.A. Sadun, C. Tonon, V. Carelli, G. Vandewalle, R. Lodi, Brain functional MRI responses to blue light stimulation in Leber's Hereditary Optic Neuropathy, Biochem. Pharmacol. (2021), 114488, https://doi.org/ 10.1016/j.bcp.2021.114488.

[14] S.A. Rahman, G.C. Brainard, C.A. Czeisler, S.W. Lockley, Spectral sensitivity of circadian phase resetting, melatonin suppression and acute alerting effects of 
intermittent light exposure, Biochem. Pharmacol. (2021), 114504, https://doi.org/ 10.1016/j.bcp.2021.114504.

[15] S. Hasan, S.K.E. Tam, R.G. Foster, V.V. Vyazovskiy, D.M. Bannerman, S.N. Peirson, Modulation of recognition memory performance by light and its relationship with cortical EEG theta and gamma activities, Biochem. Pharmacol. (2021), 114404, https://doi.org/10.1016/j.bcp.2020.114404.

[16] A. Wirz-Justice, D.J. Skene, M. Münch, The relevance of daylight for humans, Biochem. Pharmacol. (2020), 114304, https://doi.org/10.1016/j. bcp.2020.114304.

[17] C.F. Reichert, S. Veitz, M. Bühler, G. Gruber, G. Deuring, S.S. Rehm, K. Rentsch, C. Garbazza, M. Meyer, H. Slawik, Y.-S. Lin, J. Weibel, Wide awake at bedtime? Effects of caffeine on sleep and circadian timing in male adolescents - A randomized crossover trial, Biochem. Pharmacol. (2020), 114283, https://doi.org/ 10.1016/j.bcp.2020.114283.

[18] L.E. McKillop, S.P. Fisher, L. Milinski, L.B. Krone, V. V Vyazovskiy, Diazepam effects on local cortical neural activity during sleep in mice, Biochem. Pharmacol. (2021), 114515, https://doi.org/10.1016/j.bcp.2021.114515.

[19] A. Goldbeter, J.-C. Leloup, From circadian clock mechanism to sleep disorders and jet lag: Insights from a computational approach, Biochem. Pharmacol. (2021), 114482, https://doi.org/10.1016/j.bcp.2021.114482.
[20] G. Nguyen, S. Postnova, Progress in modelling of brain dynamics during anaesthesia and the role of sleep-wake circuitry, Biochem. Pharmacol. (2021), 114388, https://doi.org/10.1016/j.bcp.2020.114388.

[21] J. Taillard, P. Sagaspe, P. Philip, S. Bioulac, Sleep timing, chronotype and social jetlag: Impact on cognitive abilities and psychiatric disorders, Biochem. Pharmacol. (2021), 114438, https://doi.org/10.1016/j.bcp.2021.114438.

[22] C. Florea, P. Topalidis, T. Hauser, M. Angerer, A. Kurapov, C.A. Beltran Leon, D. Soares Brandão, M. Schabus, Sleep during COVID-19 lockdown: A cross-cultural study investigating job system relevance, Biochem. Pharmacol. (2021), 114463, https://doi.org/10.1016/j.bcp.2021.114463.

Gilles Vandewalle* GIGA-Cyclotron Research Centre-In Vivo Imaging, University of Liège, Belgium

"Address: GIGA-Cyclotron Research Centre-In Vivo Imaging, University of Liège, Bâtiment B30, 8 allée du Six août, 4000 Liège, Belgium. E-mail address: gilles.vandewalle@uliege.be. 